\title{
Detection of Advanced Neoplasia with FIT Versus Flexible Sigmoidoscopy Versus Colonoscopy: More Is More
}

\author{
Robert E. Schoen · Jorge D. Machicado
}

Published online: 21 February 2015

(c) Springer Science+Business Media New York 2015

Flexible sigmoidoscopy visualizes the rectum and the sigmoid colon and, at times, the descending colon and even the splenic flexure. Unlike colonoscopy, it does not permit inspection of the entire transverse and the right colon. As a result, the efficacy of flexible sigmoidoscopy in preventing colorectal cancer in the proximal colon is dependent on identifying abnormalities in the distal colon, which will predict neoplasia in the proximal colon. Individuals with a negative flexible sigmoidoscopy do not go on to colonoscopy, so subjects with isolated proximal neoplasia are not detected and flexible sigmoidoscopy cannot prevent colorectal cancer in that circumstance.

What criteria on flexible sigmoidoscopy should determine that a subsequent colonoscopy be performed? In order to limit and target colonoscopy use, the large, randomized trials of flexible sigmoidoscopy employed different strategies to define who should be sent for colonoscopy. In the UK Flexi-Scope trial [1], colonoscopy was performed when the following was encountered on sigmoidoscopy: (1) a polyp $\geq 10 \mathrm{~mm}$ (whether adenomatous or not), (2) $\geq 3$ adenomas, (3) an adenoma with tubulovillous or villous histology or high-grade dysplasia, or (4) a malignancy. The Italian SCORE trial [2] adopted the UK trial guidelines, but lowered the threshold to include colonoscopy for patients with an adenoma between 6 and $9 \mathrm{~mm}$. In the Norwegian NORCAPP trial [3], an even more expansive threshold was adopted, with referral to colonoscopy for any adenoma. These strategies referred approximately 5,8 , and $20 \%$ of subjects for colonoscopy,

R. E. Schoen $(\square) \cdot$ J. D. Machicado Division of Gastroenterology, Hepatology and Nutrition, University of Pittsburgh, 200 Lothrop St., Mezzanine Level, C Wing, Pittsburgh, PA 15213-2582, USA

e-mail: rschoen@pitt.edu respectively. And what about fecal immunochemical testing (FIT) — how well does it identify advanced neoplasia compared with endoscopic testing?

In this issue of Digestive Diseases and Sciences, Castro et al. [4], administered a FIT test prior to colonoscopy in subjects scheduled to undergo either screening colonoscopy [5] or colonoscopy as part of study of individuals with a family history of cancer. The authors then evaluated the detection of advanced neoplasia, defined as an adenoma $\geq 1 \mathrm{~cm}$, or with villous/tubulovillous histology or high-grade dysplasia. They compared the yield in detection of colonoscopy to FIT, and to what they estimated flexible sigmoidoscopy would have discovered using the three different criteria for referral to colonoscopy employed in the randomized trials, as outlined above [1-3]. The authors also examined a "hybrid strategy," which evaluated the yield of FIT in conjunction with flexible sigmoidoscopy.

The investigators used a standard cutoff concentration for defining a positive FIT test, $\geq 20$ microgram of hemoglobin per gram of feces $(\mu \mathrm{g} / \mathrm{g})$ [6]. A standard definition of advanced neoplasia was used, and the proximal colon was defined as proximal to the splenic flexure. Although the primary results used findings in the rectum and sigmoid colon on "simulated" sigmoidoscopy, the authors performed a sensitivity analysis and included the descending colon, but it had no impact on the results. Although half of the cohort was drawn from a trial that focused on subjects with a family history of colorectal cancer, the prevalence of advanced neoplasia was similar and the two cohorts can be looked at as a group.

Table 1 summarizes the results. At a FIT cutoff of $20 \mu \mathrm{g} / \mathrm{g}$ feces, only $6.6 \%$ of the 1,292 subjects would have been referred on for colonoscopy. The colonoscopy referral rate is greater with flexible sigmoidoscopy: $10.1 \%$ for the UK 
Table 1 Effectiveness of FIT versus various sigmoidoscopy strategies versus colonoscopy (gold standard) in detecting advanced neoplasia $(N=1,292)$

\begin{tabular}{lllll}
\hline & FIT $(\geq 20 \mu \mathrm{g} / \mathrm{g})$ & UK strategy & SCORE strategy & NORCAPP strategy \\
\hline \% referred to colonoscopy & 6.6 & 10.1 & 12.7 & 23.5 \\
\% of advanced neoplasia (AN) detected & 34.0 & 69.3 & 69.9 & 76.5 \\
\% of distal AN detected & 42.6 & 91.3 & 91.3 & 92.2 \\
\% of proximal AN detected & 17.0 & 21.3 & 23.4 & 42.5 \\
\hline
\end{tabular}

strategy, $12.7 \%$ for SCORE, and $23.5 \%$ for NORCAPP, the highest of all. What do you get for these variable rates of colonoscopy compared to the gold standard of sending everyone for colonoscopy? Overall, $11.8 \%$ of the 1292 subjects had advanced neoplasia: $8.9 \%$ in the distal colon and $3.6 \%$ in the proximal colon. Of all advanced neoplasia, FIT only detected $34 \%$, the UK and SCORE flexible sigmoidoscopy strategy would have detected close to $70 \%$, and NORCAPP would have detected $76.5 \%$ (Table 1).

Of the advanced neoplasia that would be detected, what percentage would be detected in the proximal as opposed to the distal colon? Flexible sigmoidoscopy detected $>91 \%$ of advanced neoplasia in the distal colon. This is not surprising since sigmoidoscopy involves direct inspection of the distal colon. Of the advanced proximal colon neoplasia, the UK and SCORE strategies detected 21-23\%, whereas NORCAPP detected $42.5 \%$. In contrast, FIT is not nearly as good at identifying advanced neoplasia, detecting only $42.6 \%$ of advanced distal and only $17 \%$ of advanced proximal neoplasia. The hybrid strategy where FIT is added to flexible sigmoidoscopy refers about $2-3 \%$ more people for colonoscopy with a resulting modest increase in advanced proximal neoplasia detection that is statistically not significantly different.

Flexible sigmoidoscopy is a superb means of detecting distal advanced neoplasia. In the UK [1] and the Prostate, Lung, Colorectal and Ovarian (PLCO) cancer screening trial in the USA [7], flexible sigmoidoscopy reduced colorectal cancer incidence in the distal colon by a stunning $50 \%$ compared with the control group. The threshold for referral to colonoscopy does influence the detection rate of advanced proximal neoplasia. The more liberal NORCAPP strategy, which sent anyone with an adenoma on flexible sigmoidoscopy to colonoscopy, detects $42.5 \%$ of advanced proximal neoplasia, whereas the more restrictive UK and SCORE approaches detect only 21-23\%. In the US PLCO trial, an even more liberal approach was used, with any polyp (adenoma or not, since no biopsies were performed at the time of flexible sigmoidoscopy) referred for colonoscopy. Among the randomized trials of flexible sigmoidoscopy, only the PLCO trial demonstrated a statistically significant reduction in proximal colon cancer incidence. Presumably, enough subjects were sent for colonoscopy and neoplastic polyps identified and removed in the proximal colon that a statistically significant reduction in proximal colon cancer incidence was observed.

Unfortunately, FIT is not terribly effective at detecting advanced neoplasia. At the standard threshold used in this study, FIT detected only $34 \%$ of all lesions and only $17 \%$ of those in the right colon. Previous studies have examined the effectiveness of combining FIT with sigmoidoscopy. Although a Japanese study showed that the combining FIT with sigmoidoscopy improved detection of proximal advanced neoplasia over sigmoidoscopy alone [8], adding FIT to sigmoidoscopy in the large, randomized NORCAPP trial increased the referral for colonoscopy but did not increase detection of advanced adenoma or cancer [3]. In summary, sigmoidoscopy does an excellent job in the left colon, and FIT does not add much to proximal advanced neoplasia detection.

Colonoscopy detects more advanced neoplasia than all other tests. But identifying all the subjects harboring advanced proximal neoplasia requires a lot of negative colonoscopy exams, and those exams are expensive and inconvenient. The least invasive test, FIT, is least effective. Flexible sigmoidoscopy is better than FIT. FIT plus flexible sigmoidoscopy is basically equivalent to flexible sigmoidoscopy alone. Flexible sigmoidoscopy, even with a less restrictive or more expansive rate of referral for colonoscopy, is still not as good as colonoscopy. One caveat to this hierarchy is that we are discussing detection, absent the issue of compliance. If people will not go for a colonoscopy, then it does not matter how well it performs. But, when the focus is purely on detection, the data are clear, and the old adage does not apply: less is not more-more is more.

\section{References}

1. Atkin WS, Edwards R, Kralj-Hans I, et al. Once-only flexible sigmoidoscopy screening in prevention of colorectal cancer: a multicentre randomised controlled trial. Lancet. 2010;375: $1624-1633$.

2. Segnan N, Armaroli P, Bonelli L, et al. Once-only sigmoidoscopy in colorectal cancer screening: follow-up findings of the Italian Randomized Controlled Trial-SCORE. J Natl Cancer Inst. 2011;103:1310-1322. 
3. Holme O, Loberg $M$, Kalager $M$, et al. Effect of flexible sigmoidoscopy screening on colorectal cancer incidence and mortality: a randomized clinical trial. [Erratum appears in JAMA. 2014 Sep 3;312(9):964]. JAMA 2014;312:606-615.

4. Castro I, Estevez P, Cubiella J, et al. Diagnostic performance of fecal immunochemical test and sigmoidoscopy for advanced rightsided colorectal neoplasms. Dig Dis Sci. (Epub ahead of print). doi:10.1007/s10620-014-3434-6.

5. Quintero E, Castells A, Bujanda L, et al. Colonoscopy versus fecal immunochemical testing in colorectal-cancer screening. $N$ Engl J Med. 2012;366:697-706.
6. Chiang T-H, Chuang S-L, Chen SL-S, et al. Difference in performance of fecal immunochemical tests with the same hemoglobin cutoff concentration in a nationwide colorectal cancer screening program. Gastroenterology. 2014;147:1317-1326.

7. Schoen RE, Pinsky PF, Weissfeld JL, et al. Colorectal-cancer incidence and mortality with screening flexible sigmoidoscopy. $N$ Engl J Med. 2012;366:2345-2357.

8. Kato J, Morikawa T, Kuriyama M, et al. Combination of sigmoidoscopy and a fecal immunochemical test to detect proximal colon neoplasia. Clin Gastroenterol Hepatol. 2009;7:1341-1346. 